\title{
A PROPOSAL OF PREDICTIVE MODELS FOR IMPRESSION FORMATION ${ }^{1}$
}

\author{
TOYOHIKO IWASHITA ${ }^{2}$
}

Department of Psychology, Waseda University

\begin{abstract}
In regard to the finding of Asch (1946) the interpretation was suggested that in the semantic space of individuals a quality familiar to the dimension correlating highly with $\mathrm{E}$ (evaluative) dimension, plays the 'central ' role in impression formation, while a quality familiar to the dimension correlating only slightly with $\mathrm{E}$ dimension remains 'peripheral '. Based on this interpretation, predictive models were constructed and tested for goodness of fit. These models indicate that the impression of an object is represented by the accumulation of values obtained when each quality attributed to the object is measured on the scale of a dimension correlating highly with $\mathrm{E}$ dimension. The result of the test showed a reasonable accuracy of prediction made by the suggested models.
\end{abstract}

It is Asch (1946) who attempted an experimental approach to the field of 'impression formation' from the angle of process of integrating information. He concluded that impression formation does not consist merely of a process of mosaic integration but of a specific process of organizing information attributed to an object. His viewpoint about the impression uniformity is that of the configurationists.

According to the Asch's findings, all of the character-qualities attributed to a person do not equally contribute to forming impressions. Some of them play 'central' role in the process of forming impressions and cause limitation or transformation of the other peripheral qualities and, occasionally, addition of new qualities by conjecture; it is as the result of this process that the total impression of the person is organized. For example, in his experiments the characteristics 'warm-cold 'play central roles when warm or cold is allocated in the center of the informative qualities

1 This study was conducted as one of the research projects at the NHK Radio and TV Culture Research Institute.

2 The author was a staff member of the institute until the end of March 1974.
" intelligent-skillful-industrious-determined-practical-cautious" (Series A).

However, as Asch himself points out, even in the Series A, the weight of warm or cold varies-within limits-from $S$ to $S$. In other words, though 'warm-cold' shows a tendency to play a central role as a total group result, a considerable number of independent $S$ s relegates 'warmcold' to the lower importance than to other qualities.

Asch commented that "it seemed desirable to turn first to those processes which hold generally, despite individual differences' and did not deal with the role of individual differences. However, it is inevitably necessary to obtain a knowledge of central information if we are to form a predictive model with due regard to the suggestions given by the configurationists. And this knowledge is only to be derived from the individual differences. Our suggestion is to induce the knowledge as to the $S \mathrm{~s}^{\prime}$ individual tendencies and intensities in their determination of 'central' information to predictive models. For this purpose, consistency of individual tendencies in determining ' central' information must be confirmed first of all.

Asch indicated the possibility of describing an impression construed into an evalua- 
tive dimension. When he found that impression of a person differed greatly if 'warm' was replaced with 'cold' or vice versa, he concluded that 'warm' contributes to form a positive impression, while 'cold' contributes to form a negative impression.

If this indication is agreed, the fact that a certain kind of information plays an important role in impression formation means that there is a sharp correlation between the information and the evaluative dimension of impression, which leads us to induce that our assumption of consistency of individual tendencies can also be replaced by the description that there is a high correlation between the evaluative dimension and another specific dimension in the semantic space of a person. This assumption apparently contradicts the notion taken hitherto of semantic space that the semantic space of a person consists of three independent dimensions of Evaluation (E), Potency (P) and Activity (A) (Osgood, 1957, 1962, 1964; Jakobovitz, 1966).

We have repeatedly obtained the data that show a correlation between the likedislike evaluation of an object and a characteristic of a specific dimension perceived by the $S$ as one of the attributes of the object, as well as remarkable individual differences in the perceivers' like-dislike evaluations of an object in regard to which dimension and to which direction they have a high and positive correlation (Iwashita, 1961, 1963; Onda, 1970). It has also been clarified that the semantic space obtained by Semantic Differential does not consist of three independent dimensions of $\mathrm{E}, \mathrm{P}$ and $\mathrm{A}$, but of a compound $\mathrm{E}$ and $\mathrm{P}$ dimension and an independent $\mathrm{A}$ dimension for certain kinds of $S \mathrm{~s}$, and of a compound $\mathrm{E}$ and $\mathrm{A}$ dimension and an independent $\mathrm{P}$ dimension for other kinds of $S \mathrm{~s}$ (Iwashita, 1972b; Onda, 1972). Ichihara (1971), Isogai \& Chiziiwa (1971) and Murakami (1973) also indicate a considerable correlation between the $\mathbf{E}$ dimen- sion and either of the other two dimensions, and individual differences in $S s^{\prime}$ semantic spaces.

If these facts are accepted, it is assumed, on the basis of the notion that $\mathrm{E}$ dimension is more important than the other dimensions in the semantic space (Osgood, Suci, \& Tannenbaum, 1957; Levy \& Dugan, 1960), that 'a trait which bears distinctively a meaning of a dimension compounding with $\mathrm{E}$ dimension plays a central role in impression formation of an object when the trait is one of the attributes of the object'. This assumption may be paraphrased into the statement that those who have a semantic space consisting of a compound $\mathrm{E}$ and $\mathrm{P}$ dimension and an independent $\mathrm{A}$ dimension, select a trait which bears a meaning of $\mathrm{P}$ dimension distinctively and form impressions based on the trait, while those who have a semantic space consisting of a compound $\mathrm{E}$ and $\mathrm{A}$ dimension and an independent $\mathrm{P}$ dimension, select a trait which bears a meaning of A dimension distinctively and form impressions based on the trait.

This assumption was examined by Iwashita (1971). The examination was carried out by the following procedures: (1) Selection of typical person as $S$ who expresses a preference (like or dislike) for a characteristic which bears a meaning of a specific dimension in a specific direction; (2) Construction of various stimulus sets by combining central traits with peripheral traits: central traits are to be obtained from the previous investigation of the $S$ s' structures of semantic spaces; (3) Making a request to $S$ to express his impression of each set by choosing one from several adjectives previously prepared; and (4) Inspection of the data obtained from (3) in regard to the relations among "the traits included in the stimulus sets', 'the content of $S$ 's preference' and 'the chosen adjective'.

However, when we are trying to understand impression formation as a process of integrating information, the gradual ways 
of presumption must also be considered as to how a total impression of a stimulus set containing more than two important traits can be described. In this study an attempt is made to propose three predictive models formulated on our hypothesis, and to examine the accuracy of prediction of them. Our hypothesis is as follows: The total impression of an object consisting of various traits can be predicted solely by the summation of ratings related to a dimension compounding with $\mathrm{E}$ dimension in $S$ 's affective meaning space.

\section{Proposal of Predictive Models}

Whenever plural traits are attributed to an object, they are basically grouped into the following two types: (1) in case all of them is preferable to $S$, or all of them is unpreferable to $S$; and (2) in case some of them are preferable to $S$, while the rest are unpreferable. Predictive models for each type are presented as follows:

A. in case the object belongs to type (1)

$$
\begin{gathered}
\text { when } a_{\mathrm{m} \text { ax }}=x \\
\mathrm{X}=x \\
\text { when } x-a_{\max } \geqq 1 \\
\mathrm{X}=a_{\mathrm{m} \text { ax }}+\frac{1}{n-\mathrm{I}} \sum_{i=1}^{n-1}|x|
\end{gathered}
$$

where $a_{i}(i=1,2,3, \ldots n)$ and $a_{\max }$ stand for respectively the value of each important trait rated by $S$ (values given to poles are integers of $-x$ and $x$; number of points is $2 x-1$ ), and $a$ of a trait to which $|a|$ is the largest among $n$ important traits, and where $\mathrm{X}$ stands for the grade of dislikelike of the total object of which both central and peripheral traits are attributes.

B. in case the object belongs to type (2)

$$
\mathrm{Y}=\mathrm{X}_{\mathrm{N}}+\left(\mathrm{X}_{1},-\frac{\mathrm{X}_{\mathrm{N}}}{|x|}\right)
$$

where $X_{N}$ and $X_{P}$ stand for respectively $X$ deduced from the formula [1] or [2] in regard to the traits preferred by $S$ and $\mathrm{X}$ deduced from the formula [1] or [2] in regard to the traits avoided by $S$, and where $Y$ stands for the grade of dislike-like of the object of which all traits, positively or negatively important and peripheral, are attributes.

In construction of the predictive models, the following views were adopted and the following judgment was made: (1) in the case of grasping impressions by projecting them into evaluative dimensions, summation models fit better than averaging models (Osgood et al., 1957; Fishbein \& Hunter, 1964); (2) however many traits which $S$ rates at $a_{i}$ are attributed to an object, $\sum_{i=1}^{n-1}$ $a_{i}$ of those traits deviates less than 1 point from $a_{\max }$ on the scale (Iwashita, 1972a); and (3) the value of 'pessimistic evaluative stickness', the effect of which is pointed out by Osgood et al. (1957) and Morikawa, Hatano, Shimizu, Nemoto, Sakai, and Kinoshita (1960) when plural as both positive and negative are summed up, is assumed to be $\frac{X_{N}}{|x|}$ and reduced from $\mathrm{X}_{\mathrm{r}}$.

\section{Test on the Goodness of Fit}

The experimental study was conducted in order to examine the accuracy of prediction of the proposed models. It is constructed from the three parts of Selection of Subjects (procedure I-III), Preparation of Materials (procedure IV), and Measure of Accuracy of Prediction (procedure VVII).

\section{Selection of Subjects}

I: (I) 80 songs (two-minutes long, each accompanied by an orchestra) from the music program 'Minna no Uta (Songs for Everybody)' aired by NHK Radio were recorded. (2) 3 staff members of the NHK Radio \& TV Culture Research Institute and 6 graduate students of the Waseda University were presented all 80 songs sucressively and required at each presentation of the songs to evaluate on the six 5-point bipolar scales prepared by Onda 
(1970) for measurement of music impressions. (3) When more than 3 of the 9 listeners evaluated a song on a polar point of a scale and more than 3 of the remaining listeners evaluated the song on the other polar point of the same scale, the song was selected as "concept'. "Concepts" were therefore the songs most ambiguous in the sense of polysemy.

The following 8 songs were selected in this way as 'concepts': 'Hana no Kazaguruma (Floral Pinwheel)', 'Green Green (in Japanese translation)', 'Machi (My Town)', 'Ichiban Atsui Kokoro no Uta (Song of a Glowing Heart)', 'Oyama no Taisho (King among Mates)', 'Drunten im Unterland (in Japanese translation)', 'Moeagare, Yukitachi (Flame up, Snows)', and 'Suteki na Yoru (A Wonderful Night)'.

II : (1) 600 undergraduates of the International Christian University were grouped into 30 groups of 20 each, and asked to check their impressions of the 8 "music" concepts on a total of 497 -point bipolar scales prepared by Iwashita (1972a) after each presentation of the 8 concepts. (2) Total data thus oblaincd were factor-analyzed by the Centroid method and rotated by the Varimax method. (3) Factors thus extracted were identifiable as affective evaluation, potency, activity, brightness, and general evaluation. According to this result, pure scales (Osgood et al., 1957) were sclected per factor excluding " affective evaluation'.

Pure "scales" thus selected were "relaxedtense', ' excited-calm', 'dark-bright', and ' naive-refined'.

III : (1) Each of the same 600 students was asked (i) to express his music preference by choosing an adjective (music quality) from each of the 4 pairs of adjectives ( 4 pure scales), and (ii) to select the most favorable music quality among the above chosen 4 adjectives by the method of paired comparison: that is, the most favorable quality had to be selected 3 times among the 6 combinations of the 4 qualities. (2) Each of the students was asked to express, on the 7-point scale of ' most favorable-most unfavorable', degrees of his preference as to the 8 music qualities ( 8 adjectives of the 4 pure scales). (3) The 600 students were grouped under their expressions of the most favorable qualities. Then those who rated the quality, which represented the group they belonged to, to 'most favorable', and simultancously rated opposite quality to 'most unfavorable' were selected as $S$ s. Ss in this study were, therefore, the typical persons whose preferences in music were inclined toward specific qualities.

$S$ s thus selected were as follows: "tense' prefers 13, 'relaxed' 11, 'excited' 16, 'calm' 12 , 'bright' 18 , and 'dark' 3 , totaling 73 .

\section{Preparation of Materials}

IV: (1) 6 songs were selected out of the 8 songs ( 8 concepts obtained in the procedure I) because of their ambiguities being polysemously higher than the other two. (2) 18 sets of songs were made by means of choosing 3 out of the 6 songs $(6 \times 3)$. (3) Any one of the 3 songs in a set was fixed to the center (2nd place) of the order while the other 2 songs were placed in reverse in either the 1 st or the $3 \mathrm{rd}$ place. That made $36(18 \times 2)$ sets of songs. (4) An announcement of 'Now it's time for Songs for Everybody. Let's sing together!' was added to each of the 36 sets like an actual radio program.

36 programs were thus prepared as materials.

\section{Measure of Accuracy of Prediction}

V: (1) A week after the Selection of the Subjects was conducted, $73 \mathrm{Ss}$ were requested to check on the 7-point "dislike-like' scale at each presentation of 36 materials. 2628 ratings $(73 \times 36)$ were obtained. (2) Based on the 'impressions of each song on the pure scales' of each $S$ (obtained from the procedure II) and the favorable or unfavorable music qualities of each $S$ (obtained from the procedure III), 148 ratings acquired from the materials consisting of the songs in which $S$ could cognize neither favorable nor unfavorable qualities were omitted.

There remained 2480 ratings.

VI: (1) Based on the "impressions of each song on the pure scales' (obtained from the procedure II) and music qualities of favorable or unfavorable to each $S$ (obtained from the procedure III), the 2480 ratings were examincd 
TABle 1

Accuracy of predictions

\begin{tabular}{c|c|c|c}
\hline & $\begin{array}{c}\text { Mean } \\
\text { (scale unit) }\end{array}$ & $S D$ & \multicolumn{1}{|c}{$\mathcal{N}$} \\
\hline Model 1 & .46 & .24 & 780 \\
Model 2 & .57 & .28 & 362 \\
Model 3 & .50 & .31 & 1338 \\
Total & .51 & .28 & 2480 \\
& & &
\end{tabular}

according to the suggested models and put into 3 groups. (2) At each of the 2480 ratings, $S$ 's to the 3 songs related to the favorable or unfavorable quality were counted. And these ratings of the important qualities $\left(a_{i}\right)$ were substituted for the corresponding items in the applicable predictive model (ratings of the peripheral qualities were all ignored). For instance, if a $S$ preferred 'excited', only the values on the 'excited-calm' scale rated by the $S$ were used as substitutes for the corresponding items of the predictive model. (3) Each of the 2480 ratings was paired with each of the 2480 expectants calculated by the predictive models.

Breakdown of the 2480 data by predictive models was as follows: Model [1] $\times 780$, Model [2] $\times 362$, and Model [3] $\times 1338$.

VII : (1) Absolute deviations were calculated between each pair of the 2480 ratings and expectants. (2) Means of absolute deviations of each model and of total models were calculated and regarded as indicators of accuracy of prediction. This indicator was adopted by the suggestion of Tanaka \& Iwamatsu (1968).

Accuracy of prediction of each model and total is shown in Table 1.

Table 1 shows that the discrepancy between the expectant and the actual ratings does not exceed, in all cases, one scale-unit. Accordingly, the models presented in this study are considered as showing a relatively high degree of accuracy of prediction.

\section{Discussion}

The experimental study presented here clearly indicates that each of the proposed three predictive models show enough goodness of fit. Next, there will be discussions on the comparison with other models concerning the accuracy of prediction (examination I), applicability of our models to impression formation of things other than music (examination Ir), and generalization of our models to $S$ s other than those used in the experimental study (examination III \& IV).

\section{Comparison with Other Models}

Every study on prediction of impression formation has adopted a procedure in which the predictive model is substituted for by evaluative ratings of every component of an object in order to presume a total impression of the object. It is rather difficult, therefore, to compare the result of our models with those of other models since our models require only the ratings of important qualities of an object to presume a total impression of it. Taking this point into consideration, the following examination was conducted:

I: (1) Ratings on the dislike-like scales of each song of each $S$ (obtained from the procedure II) were substituted in the extensive formula of the congruity model formulated by Triandis \& Fishbein (1963), in the simple averaging model, and in our models respectively, and 2480 expectants by each model were calculated. (2) Then absolute deviations between the pairs of expectants and actual ratings (obtained from the procedure $V$ ) were calculated by each model. Calculation of the means by each model followed.

Relative accuracy of predictions of the congruity model, the simple averaging model, and our models is shown in Table 2. This table presents the congruity model is higher in its accuracy of prediction than the simple averaging model $(t=4.720, d f$ $=2479, p<.001$ ), and our model is higher than the congruity model $(t=21.252, d f=$ $2479, p<.001$ ).

Table 3, which shows the comparison of accuracy of prediction among our models 
TABle 2

Relative accuracy of predictions

\begin{tabular}{l|c|c|c|c}
\hline Modelt & $\begin{array}{c}\text { Mean } \\
\text { (scale unit) }\end{array}$ & $S D$ & $\gamma$ & $\mathcal{N}$ \\
\hline S A & .64 & .38 & & 2480 \\
CM & .61 & .33 & .61 & 2480 \\
OM $\dagger$. & .50 & .30 & .67 & 2480 \\
\hline
\end{tabular}

$+\mathrm{CM}, \mathrm{SA}$ and OM refers to the congruity model, the simple averaging model and our models respectively.

忛 Figures in the column of $\mathrm{OM}$ are means of total for Models 1,2 and 3.

TABle 3

Comparison of accuracy of predictions of our models between the two cases

\begin{tabular}{c|c|c|c|c}
\hline & Mean & $S D$ & $\gamma$ & $\mathcal{N}$ \\
\hline Case 1 + & .51 & .28 & .71 & 2480 \\
Case 2 + & .50 & .30 & &
\end{tabular}

$\uparrow$ in case ratings of meaningfulness of important qualities only were applied (i.c. Table 1, column Total).

t in case evaluative ratings of all components were applied (i.e. Table 2, column OM).

shown in Table 2 and in the column of total of Table 1, brings out the interesting fact that the Case 2 is significantly higher than the Case $1 \quad(t=2.248, d f=2479, p<.05)$. This significant difference is due to the " error" caused in the prediction of evaluation of an object when only the ratings of important qualities of the object are applied.

\section{Extension to Other Materials}

Many of the studies on prediction of impression formation have used verbal information as stimuli. Accordingly, our next examination was to measure the accuracy of prediction when materials other than the music were used.

II: (1) Based on the 'impressions of each song on the pure scales' (abtained from the procedure II), a list of traits was drawn in regard to each song for each $S$.
(2) 18 programs were newly chosen from the programs presented in the procedure $V$ (those two programs which contain the same songs in different orders were regarded as the same program and either one of them was omitted), and each of the 18 programs was replaced by the list of traits. The following is an example of a program replaced by a list of traits. (3) A week after the procedure $V$ was done, $73 S$ s were presented these new 18 different sets of materials and requested to check them off on the 7-point 'dislike-like' scale in regard to each list of traits. In this way 1314 ratings were obtained $(73 \times 18)$. (4) 74 ratings acquired from the program constructed from the songs in which $S$ could cognize neither favorable nor unfavorable qualities were omitted. The remaining 1240 ratings were subjected to the same treatment used in the procedures VI and VII.

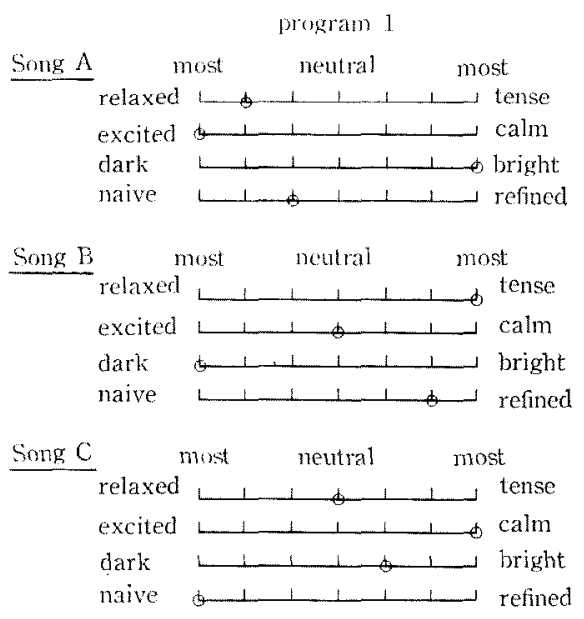

Table 4 presents the accuracy of prediction of each model and their means, with Table 1 for comparison. As is shown in this table the accuracy of prediction by each model is lightly lower than in the case where the actual programs were used but the differences are not significant (Model 1: $t=1.245, \quad d f=1166, p>.20$; Model 2: $t=1.078, d f=540, p>.20 ;$ Model 
TABLE 4

Comparison of accuracy between the two materials

\begin{tabular}{r|rrr|rrr|r}
\hline & \multicolumn{3}{|c|}{$\begin{array}{c}\text { Lists of program } \\
\text { traits }\end{array}$} & \multicolumn{3}{|c}{ Real program } \\
\cline { 2 - 7 } & Mean & $S D$ & $\mathcal{N}$ & Mean & $S D$ & $\mathcal{N}$ \\
\hline Model 1 & .48 & .35 & 388 & .46 & .24 & 780 \\
Model 2 & .60 & .35 & 180 & .57 & .28 & 362 \\
Model 3 & .51 & .38 & 672 & .50 & .31 & 1338 \\
\hline Total & .53 & .36 & 1240 & .51 & .28 & 2480
\end{tabular}

$3: t=0.630, d f=2008, p>.50$; Total: $t=$ $1.867, d f=3718, p>.05)$. The proposed three models are confirmed to be applicable to the impression formation of verbal information such as list of adjectives as well as that of actual cognitive objects such as appreciation of the music.

\section{Extension to Other Ss}

$S$ s selected for the experimental study were those typical persons whose preferences were inclined to specific qualities. Investigation of typical persons is one of the methods applied to the study of human beings when a scientific experiment, which attempts to extract a factor from complex factors of a phenomenon without converting the other factors in order to examine its function, was introduced to this field. This method creates a more precise control over conditions so that the image of living human beings should not be artificially distorted. However, it may be argued that these typical persons are exceptions and findings deduced from their responses are not to be generalized. In answer to this argument the following examination was carried out.

III: (1) Among the 600 students, 515 persons were newly selected on the first one of the following two standards given in the procedure III for selection of $S \mathrm{~s}$ : (i) $S$ should be those who select one music quality with a frequency of 3 times in the method of paired comparison of the 4 qualities; and at the same time (ii) $S$
Table 5

Comparison of accuracy between the two groups of $S$ s (1)

\begin{tabular}{r|rrr|rrr}
\hline & \multicolumn{3}{|c|}{ Expansive $S \mathrm{~s}$} & \multicolumn{3}{c}{ Typical $S \mathrm{~s}$} \\
\cline { 3 - 7 } & Mean & $S D$ & $\mathcal{N}$ & Mean & $S D$ & $\mathcal{N}$ \\
\hline Model 1 & 1.04 & .49 & 214 & .46 & .24 & 780 \\
Model 2 & .54 & .36 & 456 & .57 & .28 & 362 \\
Model 3 & .52 & .33 & 1004 & .50 & .31 & 1338 \\
\hline Total & .70 & .39 & 1674 & .51 & .28 & 2480
\end{tabular}

should be those who manifest ' most favorable' to the above selected quality and ' most unfavorable' to the opposite quality. This group of 515 amounted to $85.8 \%$ of the 600 students. (2) 73 persons whose data had been already used in the experimental study were excluded. From the remaining 442, 50 persons were randomly selected as $S$ s for this examination. (3) The $50 \mathrm{~S}$ s were subjected to the same treatment used in the procedure $\mathrm{V}$, and 188 ratings were obtained. (4) 126 ratings were omitted because they were obtained from the programs containing the songs in which $S$ could cognize neither favorable nor unfavorable qualities. Then the remaining 1674 ratings were subjected to the same treatment used in the procedures VI and VII. Accuracy of predictions by each predictive model and the total obtained from the above ratings is shown in Table 5 with attachment of Table 1 for comparison. This table indicates that the accuracy of predictions of the total of Models 1,2 and 3 is lower than that of the case in which the typical persons were used $(t=18.267$, $d f=4152, p<.001)$. However, the reason is clear in the table itself. In Model 1, there is a significant difference between the two groups of $S \mathrm{~s}(t=24.096, d f=992, p<$ $.001)$, while there are no significant differences either in Model $2(t=1.302, d f=$ $816, p>10)$ or in Model $3(t=1.518, d f=$ $2340, p>.10)$. What is then the cause of this decrease in accuracy of prediction in the case to which Model I was applied?

Model 1, as was stated in the section of 
Proposal of Predictive Models, was established to be applied when all of the components attributed to an object are either of favorable qualities or unfavorable qualities, and at the same time they are rated on the polar point of the scale related to the important qualities. Our hypothesis on this model is, that the evaluative value of an object in such a case, is represented by the scale values of the polar points. However, in the case of the examination III, the ratings applicable to this model were relatively few in number, and in cases they were applied, observations of evaluation to the object tended to fall short of the expectants. On the other hand, in the ratings applicable to the Models 2 and 3, the number of which increased according to the decrease of applicability of the ratings to the Model 1 , no consistent tendency of observations being lower than cxpectants was shown.

These results lead us to assume that those who do not show characteristic preference for an object hardly perceive a specific quality in the object, and, hardly have a specific attitude of like or dislike toward the object. On this assumption, that such characteristics of $S \mathrm{~s}$ in the examination III made the accuracy of Model 1 low, the following examination was executed.

IV: (1) Among the 600 students participated in the procedure III, those who did not satisfy the condition of selection of the most favorable quality, but submitted data of impression ratings without omission concerning the 8 songs described in the procedure II, were selected as $S$ s. There were 42 such $S$ s. (2) 1512 ratings were obtained from these $S$ s by conducting the same treatment used in the procedure $\mathrm{V}$. (3) According to the same treatment used in the procedure $\mathrm{V}, 116$ ratings obtained from the programs containing the songs in which $S$ could cognize neither favorable nor unfavorable qualities were omitted. The remaining 1396 ratings were subjected to the same treatment used in the
TABle 6

Comparison of accuracy between the two groups of $S$ s (2)

\begin{tabular}{r|rrr|rrr}
\hline & \multicolumn{2}{|c|}{ More expansive $S \mathrm{~s}$} & \multicolumn{4}{|c}{ Expansive $S \mathrm{~s}$} \\
& Mean & $S D$ & \multicolumn{1}{c|}{$\mathcal{N}$} & Mean & $S D$ & $\mathcal{N}$ \\
\hline Model 1 & 1.16 & .58 & 112 & 1.04 & .49 & 214 \\
Model 2 & .56 & .35 & 426 & .60 & .36 & 456 \\
Model 3 & .51 & .31 & 858 & .52 & .33 & 1004 \\
\hline Total & .74 & .41 & 1396 & .72 & .39 & 1674
\end{tabular}

procedures VI and VII.

Table 6 shows the result of the above examination with attachment of the result of procedure $\mathrm{V}$ for comparison. Comparison between the results indicates that there are no significant differences between the two groups of $S \mathrm{~s}$ in any case of Total, the mean of Model 1,2 and 3, $(t=1.382$, $d f=3068, p>.10)$, Model $2(t=0.835, d f$ $=880, p>.40)$ and Model $3(t=0.670$, $d f=1860, p>.50)$ but the goodness of fit of the Model 1 decreased with significance. In other words, it is clear that the more ambiguous $S$ 's preference is the lower the goodness of fit of Model 1 becomes.

Ambiguity in preference for an object, however, means meagerness of ego-involvement with the object. Accordingly, there is no necessity nor inevitability for a person to form an impression of such an object. If one is asked to form an impression of such an object it must be rather like solving a puzzle for him. To understand the human behavior in such a situation, for which a special investigation may be required, is irrelevant to the purpose of the present study. Consequently, despite the decrease in the accuracy of prediction of Model 1 as was shown in the section of Extension to Other $S \mathrm{~s}$, the proposed three models are inferred to be of sufficient generality in so far as they are concerned to the mechanisms of impression formation caused by the spontaneous human behavior. 


\section{REFERENCES}

Asch, S. E. 1946 Forming impressions of personality. Journal of Abnormal and Social Psychology, 41, 258-290.

Fisheern, M., \& Hunter, R. 1964 Summation versus balance in attitude organization and change. Journal of Abnormal and Social Psychology, 69, 505-510.

ICHHARA, Y. 1971 Psychological study of appreciation of pictures (IV). Tokyo Toritsu Daigaku Jinbun Gakuho, 83, 53-102 (in Japanese).

Isogar, Y., \& Chizirwa, H. 1971 A psychological study on evaluation and appreciation of pictures. Bulletin of the Musashino Art University, 5, 34-58 (in Japanese with English Summary).

IWASHIT, T, 1961 A basic study of the mechanisms of interpersonal feeling and of its perception (1). The Japanese Journal of Psychology, 32, 84-96 (in Japanese).

IwashitA, T. 1963 A Basic study of the mechanisms of interpersonal feeling and of its perception (2). The Japanese Journal of Psychology, 34, 59-73 (in Japanese).

Iwashita, T, 1971 A basic study on the mechanism of impression formation. Waseda Psychological Reports, 3, 24-31 (in Japanese).

Iwashita, T. $1972 \mathrm{a}$ How to understand the mechanism of program perception of audience. The Annual Bulletin of NHK Radio \& TV Culture Research, 17, 222-254 (in Japanese).

Iwashrra, T. $1972 \mathrm{~b}$ An experimental study on the individual differences of affective meaning space. The Japanese Fournal of Psychology, 43, 188-200 (in Japanese with English Summary).

Jakoвovitz, L. 1966 Comparative psycholinguistics in the study of cultures. International Journal of Psychology, 1, 2-37.
LeVY, L. H., \& Dugan, R. D. 1960 A constant error approach to the study of dimensions of social perception. Journal of Abnomal and Social Psychology, 61, 21-24.

Morikawa, Y., Hatano, T., Shimize, M., Nemoto, N., Sasaki, T., \& Kinoshita, T. 1960 Quantitative study of meaning. Japanese Psychological Review, 4, 61-84 (in Japanese).

Murakami, Y. 1973 An experimental study of semantic differential method. The Japanese Journal of Psychology, 44, 179-185 (in Japanese with English Summary).

ONDA, N. 1970 An experimental study on the process of forming impression. The $N H K$ Report on Broadcast Research, 1970.10, 34-45 (in Japanese).

Onda, N. 1972 A study on the affective meaning space. The Annual Bulletin of NHK Radio 8 TV Culture Research, 17, 255-271 (in Japanese).

Oscood, C.E. 1957 Motivational dynamics of language behavior. In M. R. Jones (Ed.), Nebraska symposium on motivation. University of Nebraska Press, 348-424.

Osgoon, C. E. 1962 Studies on the generality of affective meaning system. American Psychologist, 17, 10-28.

OsGoon, C. E. 1964 Semantic differential technique in the comparative study of cultures. American Anthropologist, 66, 171-200.

Osgood, C. E., Suci, G. J., \& Tannenbaum, P. H. 1957 The measurement of meaning. Urbana, University of Illinois Press.

Tanaka, Y., \& Iwamatsu, Yoko. 1968 Psycholinguistic studies in cross-cultural generality in cognitive interaction. Japanese Psychological Research, 10, 45-58.

Triandrs, H. C. \& Frshrein, M. 1963 Cognitive interaction in person perception. Joumal of Abnormal and Social Psychology, 67, 446-453.

(Received Oct. 18, 1974) 